\title{
Assessment of Knowledge of Primary Health Care Providers about Routine Care of Type 2 Diabetic Patients in Primary Health Care Units in Zagazig District, Sharqia Governorate
}

\author{
Amany Mahmoud Oraby Mohammed*1, Gehan Abdel Qader Ibrahim², Hanaa Salah Said ${ }^{1}$ \\ Departments of ${ }^{1}$ Family Medicine and ${ }^{2}$ Internal Medicine, Faculty of Medicine - Zagazig University, Egypt. \\ *Corresponding author: Amany Mahmoud Oraby Mohammed, Mobile: (+20)01019520580, \\ Email: amanyoraby20@yahoo.com
}

\begin{abstract}
Background: Diabetes mellitus is a major and growing health problem in Egypt. Despite considerable advances in diagnosis and management of diabetes, there is a large gap between ideal treatment goals and actual outcomes. Lack of up-to-date knowledge, wrong attitudes and malpractice among healthcare workers regarding diabetes control may play an important role in failure to achieve therapeutic goals.

Objective: This study aimed to reduce complications and improve outcome of type 2 diabetic patients attending family medicine units \& centers through assessing the knowledge of healthcare providers about routine care of type 2 diabetes mellitus.

Patients and Methods: This cross-sectional study was carried out on 102 doctors (family physicians \& general practitioners) from Family Health Centers \& Units in Zagazig Health District, Sharqia Governorate. Data were collected through a questionnaire, which assess doctors' knowledge about type 2 diabetes mellitus. Data was collected and analyzed. Bar charts for knowledge were done.

Results: This study showed that $66.7 \%$ of doctors had good knowledge about routine care of type 2 diabetic patients.

Conclusion: The doctors were good in communication skills with patients, describing medications, emphasizing on blood sugar control.
\end{abstract}

Keywords: Healthcare provider, practical performance, Type 2 diabetes.

\section{INTRODUCTION}

Diabetes mellitus care is complex and requires patients to take an active role in the management of their disease. Currently, adequate and continuing medical care aiming at preventing acute complications, diminishing risk of long-term complications as well as patient self-management education are considered standard in the care for type 2 diabetes patients ${ }^{(\mathbf{1})}$.

The management of DM largely depends on the patient's ability to do self-care in their daily lives, and therefore, patient education is always considered an essential element of DM management. Studies have shown that patients, who are knowledgeable about the DM self-care, have better long-term glycemic control ${ }^{(2)}$.

Knowledge about glycemic control can help the people to understand the risk of diabetes and motivate them to seek proper treatment and care and to keep the disease under control ${ }^{(3)}$. This study aimed to reduce complications and improve outcome of type 2 diabetic patients attending family medicine units \& centers through assessing the knowledge of healthcare providers about routine care of type 2 diabetic patients attending family health centers \& units.

\section{PATIENTS AND METHODS}

This cross-sectional study was carried out on 102 doctors (family physicians \& general practitioners) from Family Health Centers \& Units in Zagazig Health District, Sharqia Governorate from March 2019 to March 2021. They were selected randomly to represent Sharqia Governorate (which administratively divided into 17 major cities according to Sharkia.gov.eg, 2018). The study was conducted in the family health centers and units (accredited and not accredited), which affiliate to Zagazig health district.

Inclusion criteria were; Primary health care providers (general practitioner or family physician) who are working in primary health care units in Zagazig district. The data were collected by using American Diabetes Association up to date recommendations for routine care of type 2 DM in 2018, a structured questionnaire was used to assess physicians' knowledge and checklist to assess their practice.

\section{Operational Design:}

Fieldwork started at the beginning of March 2019, and completed by the end of March 2021 by attending the health facilities 3 days/ week. The visits to each selected center was done at different days in order to ensure complete week coverage.

The observation was done after taking permission from doctors without clearing the actual aim of the visit (assess doctors performance) to avoid their trials to improve his/her performance during observation. It wasn't ethically and should not happen but many authors do this for the same reasons provided taking permission from higher authorities ${ }^{(4)}$.

The researcher start to fill the items of personal data $\&$ knowledge by asking doctors then fill the rest of the sheet away from them with putting in consideration that the researcher in the field cannot rely on their 
memory, so it was essential that observations captured in clear, detailed, and descriptive notes.

All subjects included in this study were interviewed and the questionnaires were filled by the researcher, which took about 10 minutes and chick list took about 20-30 minutes.

\section{Ethical consent:}

An approval of the study was obtained from Zagazig University Academic and Ethical Committee. Every patient signed an informed written consent for acceptance of the study.

This work has been carried out in accordance with The Code of Ethics of the World Medical Association (Declaration of Helsinki) for studies involving humans.

\section{Statistical analysis}

The collected data were coded, processed and analyzed using the SPSS (Statistical Package for Social Sciences) version 22 for Windows ${ }^{\circledR}$ (IBM SPSS Inc, Chicago, IL, USA). Data were tested for normal distribution using the Shapiro Walk test. Qualitative data were represented as frequencies and relative percentages.

Chi square test $(\chi 2)$ was used to calculate difference between two or more groups of qualitative variables. Quantitative data were expressed as mean \pm SD. Independent samples t-test was used to compare between two independent groups of normally distributed variables (parametric data). $\mathrm{P}$ value $\leq 0.05$ was considered significant.

\section{RESULTS}

Table (1): Socio demographic and personal characteristics of primary health care providers $(\mathrm{n}=102)$

\begin{tabular}{|c|c|c|}
\hline Characteristics & \multicolumn{2}{|c|}{ Value } \\
\hline $\begin{array}{l}\text { Age (years): } \\
\text { Mean } \pm \text { SD } \\
\text { Median }\end{array}$ & \multicolumn{2}{|c|}{$\begin{array}{c}33.30 \pm 5.15 \\
33\end{array}$} \\
\hline & No & $\%$ \\
\hline $\begin{array}{l}\text { Sex } \\
\text { - Male } \\
\text { - Female }\end{array}$ & $\begin{array}{c}9 \\
93\end{array}$ & $\begin{array}{c}8.8 \\
91.2\end{array}$ \\
\hline $\begin{array}{l}\text { Courses } \\
\text { - None } \\
\text { - TOEFL } \\
\text { - ICDL } \\
\text { - First aid } \\
\text { - IMCI } \\
\end{array}$ & $\begin{array}{c}47 \\
30 \\
3 \\
2 \\
20 \\
\end{array}$ & $\begin{array}{c}46.1 \\
29.4 \\
2.9 \\
2 \\
19.6\end{array}$ \\
\hline $\begin{array}{l}\text { Scientific degree } \\
\text { - General practitioner } \\
\text { - Family medicine specialists }(\mathrm{n}=66) \\
\text { Diploma } \\
\text { Master } \\
\text { Fellowship } \\
\text { Master \& Fellowship } \\
\end{array}$ & $\begin{array}{c}36 \\
\\
24 \\
32 \\
7 \\
3 \\
\end{array}$ & $\begin{array}{c}35.3 \\
\\
23.5 \\
31.4 \\
6.9 \\
2.9 \\
\end{array}$ \\
\hline $\begin{array}{l}\text { Years of experience } \\
-<2 \\
-2-4 \\
\text { - }>4\end{array}$ & $\begin{array}{l}10 \\
26 \\
66\end{array}$ & $\begin{array}{c}9.8 \\
25.5 \\
64.7\end{array}$ \\
\hline \begin{tabular}{|l|} 
No. of nurseslassistants working with you \\
-1 \\
-2
\end{tabular} & $\begin{array}{l}91 \\
11 \\
\end{array}$ & $\begin{array}{l}89.2 \\
10.8 \\
\end{array}$ \\
\hline
\end{tabular}

This study showed that the mean age of primary health care providers was $33.30 \pm 5.15$ years and the highest percentage of them were females, without courses, general practitioner as a last degree \& had $>4$ years of experience, 1 nurseslassistants working with them, and communication skills with percent $(91.2 \%, 46.1 \%, 35.3 \%, 64.7,89.2 \%$ \& $67.6 \%$ respectively) as shown in table (1). Figure (1) showed that about two thirds $(66.7 \%)$ of primary health care providers had inadequate total knowledge score about routine care of type 2 diabetes mellitus. 


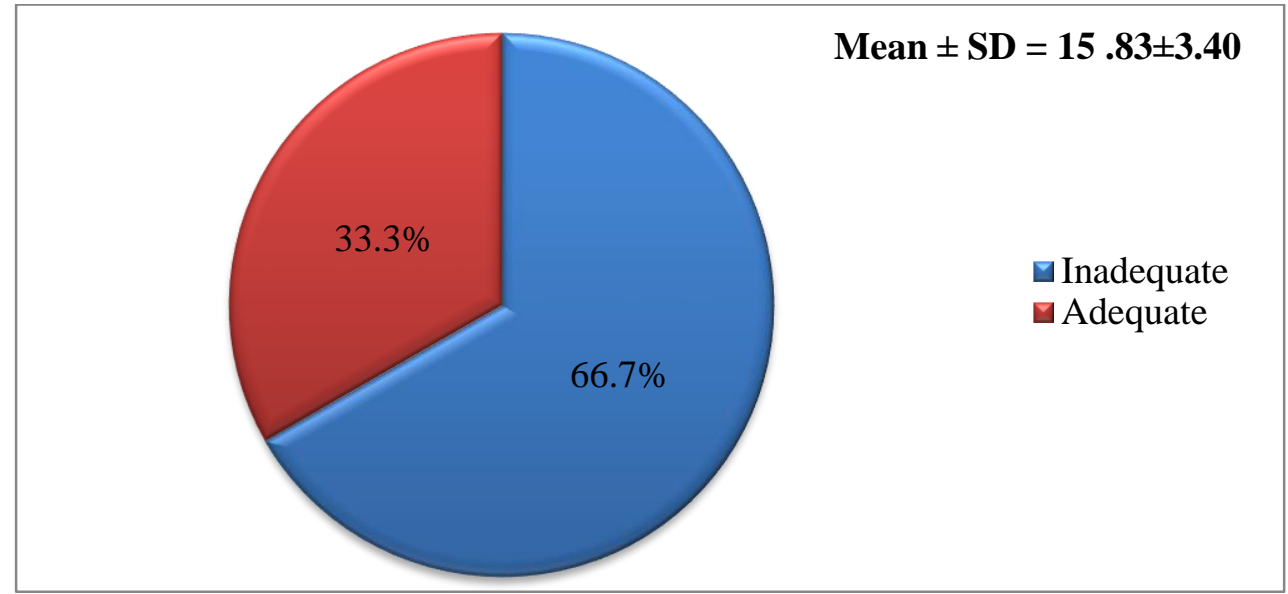

Figure (1): Total score of knowledge of primary health care providers about routine care of type 2 diabetes mellitus $(n=102)$.

Figure (2) showed that there was statistically significance difference $\left(\mathrm{P}<0.05^{*}\right)$ between family physicians and general practitioners regarding total knowledge score about routine care of type 2 diabetes mellitus.

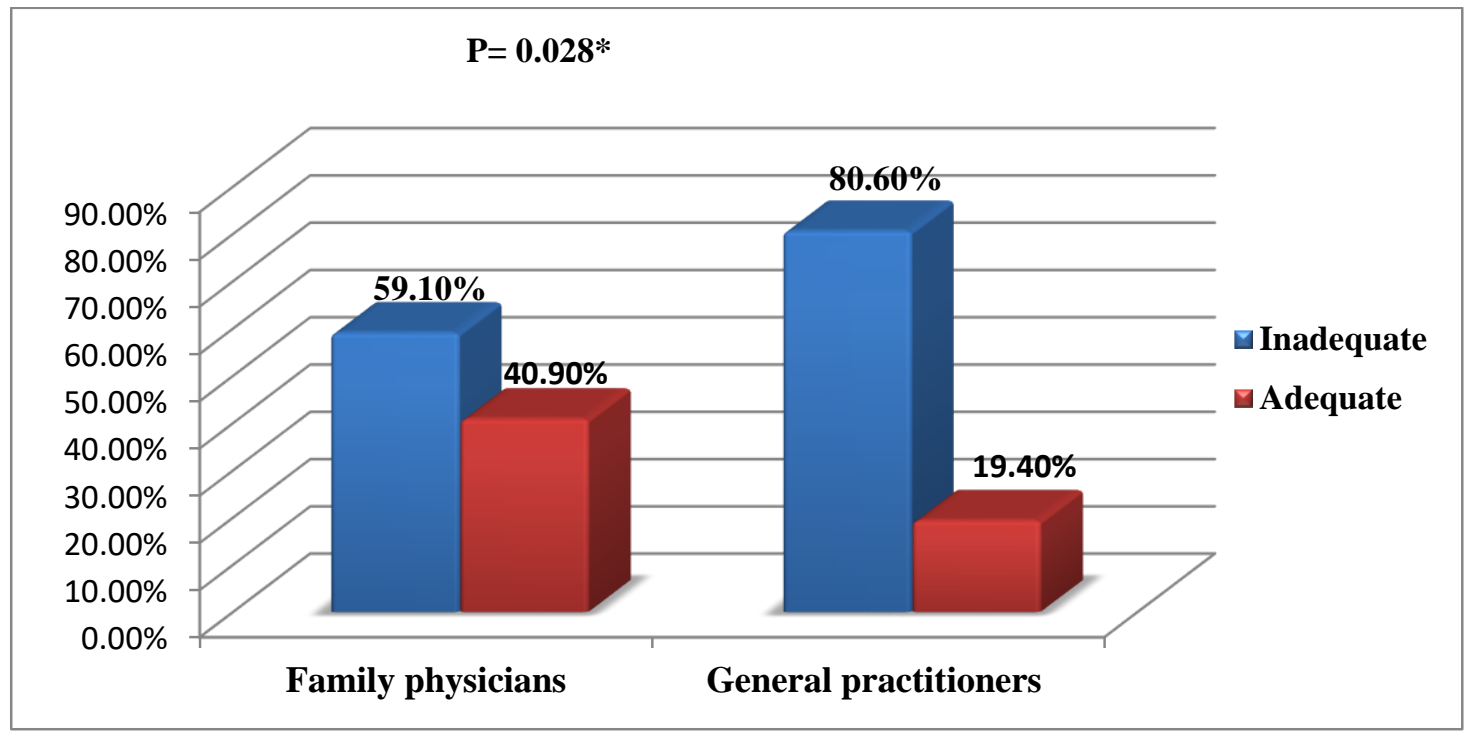

Figure (2): Comparison between family physicians and general practitioners regarding total score of knowledge about routine care of type 2 diabetes mellitus ( $\mathrm{n}=102)$.

Table (2): Comparison between family physicians and general practitioners regarding knowledge about routine care of type 2 diabetes mellitus $(n=102)$

\begin{tabular}{|c|c|c|c|c|c|c|}
\hline \multirow[t]{2}{*}{ Knowledge } & \multicolumn{2}{|c|}{$\begin{array}{c}\text { Family } \\
\text { physicians } \\
(\mathbf{n}=66)\end{array}$} & \multicolumn{2}{|c|}{$\begin{array}{c}\text { General } \\
\text { practitioners } \\
(\mathbf{n}=36)\end{array}$} & \multirow[t]{2}{*}{ Test } & \multirow[t]{2}{*}{$P$ value } \\
\hline & No & $\%$ & No & $\%$ & & \\
\hline $\begin{array}{l}\text { Routine care of diabetic patients } \\
\text { - Incorrect answer (9) } \\
\text { - Correct answer (93) }\end{array}$ & $\begin{array}{c}1 \\
65\end{array}$ & $\begin{array}{l}11.1 \\
69.9\end{array}$ & $\begin{array}{c}8 \\
28 \\
\end{array}$ & $\begin{array}{l}88.9 \\
30.1\end{array}$ & ${ }^{\mathrm{a}}$ Fisher & $<0.001 * *$ \\
\hline $\begin{array}{l}\text { What meant by HbA1c } \\
\text { - Incorrect answer }(0) \\
\text { - Correct answer }(102)\end{array}$ & $\begin{array}{c}0.0 \\
66\end{array}$ & $\begin{array}{c}00 \\
64.7\end{array}$ & $\begin{array}{c}0.0 \\
36\end{array}$ & $\begin{array}{c}00 \\
35.3\end{array}$ & - & - \\
\hline $\begin{array}{l}\text { Optimal HbA1c level diabetic patients } \\
\text { - Incorrect answer (15) } \\
\text { - Correct answer (87) }\end{array}$ & $\begin{array}{c}3 \\
63\end{array}$ & $\begin{array}{c}20 \\
72.4\end{array}$ & $\begin{array}{l}12 \\
24\end{array}$ & $\begin{array}{c}80 \\
27.6\end{array}$ & b15.39 & $<0.001 * *$ \\
\hline $\begin{array}{l}\text { When blood pressure assessed } \\
\text { - Incorrect answer (11) } \\
\text { - Correct answer (91) }\end{array}$ & $\begin{array}{c}2 \\
64\end{array}$ & $\begin{array}{l}18.1 \\
70.3\end{array}$ & $\begin{array}{c}9 \\
27\end{array}$ & $\begin{array}{l}81.9 \\
29.7\end{array}$ & ${ }^{\mathrm{a} F i s h e r}$ & $0.001 *$ \\
\hline Optimal level of bl. pressure for diabetic patients & & & & & ${ }^{\mathrm{a}}$ Fisher & $<0.001 * *$ \\
\hline
\end{tabular}




\begin{tabular}{|c|c|c|c|c|c|c|}
\hline \multirow[t]{2}{*}{ Knowledge } & \multicolumn{2}{|c|}{$\begin{array}{c}\text { Family } \\
\text { physicians } \\
(\mathbf{n}=66)\end{array}$} & \multicolumn{2}{|c|}{$\begin{array}{c}\text { General } \\
\text { practitioners } \\
(\mathbf{n}=36)\end{array}$} & \multirow[t]{2}{*}{ Test } & \multirow[t]{2}{*}{$P$ value } \\
\hline & No & $\%$ & No & $\%$ & & \\
\hline $\begin{array}{l}\text { - Incorrect answer (13) } \\
\text { - Correct answer (89) }\end{array}$ & $\begin{array}{c}1 \\
65\end{array}$ & $\begin{array}{l}7.7 \\
73\end{array}$ & $\begin{array}{l}12 \\
24\end{array}$ & $\begin{array}{c}92.3 \\
27\end{array}$ & & \\
\hline $\begin{array}{l}\text { Times of ordering lipid profile } \\
\text { - Incorrect answer (12) } \\
\text { - Correct answer (90) } \\
\end{array}$ & $\begin{array}{c}2 \\
64 \\
\end{array}$ & $\begin{array}{c}16.7 \\
71\end{array}$ & $\begin{array}{l}10 \\
26 \\
\end{array}$ & $\begin{array}{c}83.3 \\
29\end{array}$ & ${ }^{\mathrm{a}}$ Fisher & $<0.001 * *$ \\
\hline $\begin{array}{l}\text { Optimal level of lipid profile for diabetic patients } \\
\text { - Incorrect answer (17) } \\
\text { - Correct answer (85) }\end{array}$ & $\begin{array}{c}5 \\
61\end{array}$ & $\begin{array}{l}29.4 \\
71.8\end{array}$ & $\begin{array}{l}12 \\
24\end{array}$ & $\begin{array}{l}70.6 \\
28.2\end{array}$ & ${ }^{\mathrm{b}} 11.12$ & $0.001^{*}$ \\
\hline $\begin{array}{l}\text { How many times to assess BMI } \\
\text { - Incorrect answer (19) } \\
\text { - Correct answer (83) }\end{array}$ & $\begin{array}{c}4 \\
62 \\
\end{array}$ & $\begin{array}{c}21 \\
74.7\end{array}$ & $\begin{array}{l}15 \\
21\end{array}$ & $\begin{array}{c}79 \\
25.3\end{array}$ & ${ }^{\mathrm{b}} 19.48$ & $<0.001 * *$ \\
\hline $\begin{array}{l}\text { Optimal level of BMI for diabetic patients } \\
\text { - Incorrect answer (20) } \\
\text { - Correct answer }(82)\end{array}$ & $\begin{array}{c}9 \\
57\end{array}$ & $\begin{array}{c}45 \\
69.5\end{array}$ & $\begin{array}{l}11 \\
25\end{array}$ & $\begin{array}{c}55 \\
30.5\end{array}$ & $\mathrm{~b}_{4} 4.23$ & $0.040^{*}$ \\
\hline $\begin{array}{l}\text { Target bl. glucose level } \\
\text { - Incorrect answer (29) } \\
\text { - Correct answer (73) }\end{array}$ & $\begin{array}{l}17 \\
49 \\
\end{array}$ & $\begin{array}{c}58.6 \\
67\end{array}$ & $\begin{array}{l}12 \\
24 \\
\end{array}$ & $\begin{array}{c}41.4 \\
33\end{array}$ & b0.657 & 0.418 \\
\hline $\begin{array}{l}\text { Time of ordering glucose level } \\
\text { - Incorrect answer (34) } \\
\text { - Correct answer (68) } \\
\end{array}$ & $\begin{array}{l}24 \\
42 \\
\end{array}$ & $\begin{array}{l}70.6 \\
61.8 \\
\end{array}$ & $\begin{array}{l}10 \\
26 \\
\end{array}$ & $\begin{array}{l}29.4 \\
38.2 \\
\end{array}$ & b0.773 & 0.379 \\
\hline $\begin{array}{l}\text { Times of doing fundus examination } \\
\text { - Incorrect answer (17) } \\
\text { - Correct answer (85) } \\
\end{array}$ & $\begin{array}{c}8 \\
58 \\
\end{array}$ & $\begin{array}{l}47 \\
68 \\
\end{array}$ & $\begin{array}{c}9 \\
27 \\
\end{array}$ & $\begin{array}{l}53 \\
32 \\
\end{array}$ & b2.782 & 0.095 \\
\hline $\begin{array}{l}\text { Method to check nephropathy } \\
\text { - Incorrect answer (12) } \\
\text { - Correct answer (90) }\end{array}$ & $\begin{array}{c}3 \\
63 \\
\end{array}$ & $\begin{array}{l}25 \\
70 \\
\end{array}$ & $\begin{array}{c}9 \\
27 \\
\end{array}$ & $\begin{array}{l}75 \\
30 \\
\end{array}$ & b9.389 & $0.002^{*}$ \\
\hline $\begin{array}{l}\text { Time of ordering nephropathy check } \\
\text { - Incorrect answer (10) } \\
\text { - Correct answer (92) }\end{array}$ & $\begin{array}{c}2 \\
64 \\
\end{array}$ & $\begin{array}{l}20 \\
70\end{array}$ & $\begin{array}{c}8 \\
28 \\
\end{array}$ & $\begin{array}{l}80 \\
30 \\
\end{array}$ & ${ }^{\mathrm{a}}$ Fisher & $0.002^{*}$ \\
\hline $\begin{array}{l}\text { If vaccinations recommended to diabetic patients } \\
\text { - Incorrect answer (14) } \\
\text { - Correct answer (88) }\end{array}$ & $\begin{array}{c}4 \\
62\end{array}$ & $\begin{array}{l}28.6 \\
70.5\end{array}$ & $\begin{array}{l}10 \\
26\end{array}$ & $\begin{array}{l}71.4 \\
29.5\end{array}$ & ${ }^{\mathrm{a}}$ Fisher & $0.002^{*}$ \\
\hline $\begin{array}{l}\text { Type of recommended vaccines } \\
\text { - Incorrect answer (23) } \\
\text { - Correct answer (79) }\end{array}$ & $\begin{array}{c}8 \\
58 \\
\end{array}$ & $\begin{array}{c}35 \\
73.4 \\
\end{array}$ & $\begin{array}{l}15 \\
21\end{array}$ & $\begin{array}{c}65 \\
26.6 \\
\end{array}$ & ${ }^{\mathrm{b}} 11.64$ & $0.001 *$ \\
\hline $\begin{array}{l}\text { Times to check feet of patients } \\
\text { - Incorrect answer (45) } \\
\text { - Correct answer (57) }\end{array}$ & $\begin{array}{l}30 \\
36 \\
\end{array}$ & $\begin{array}{c}66.7 \\
63 \\
\end{array}$ & $\begin{array}{l}15 \\
21 \\
\end{array}$ & $\begin{array}{c}33.3 \\
37\end{array}$ & b0.136 & 0.713 \\
\hline $\begin{array}{l}\text { How can you examine feet } \\
\text { - Incorrect answer (12) } \\
\text { - Correct answer (90) }\end{array}$ & $\begin{array}{c}2 \\
64 \\
\end{array}$ & $\begin{array}{c}16.7 \\
71\end{array}$ & $\begin{array}{l}10 \\
26 \\
\end{array}$ & $\begin{array}{c}83.3 \\
29\end{array}$ & ${ }^{\mathrm{a}}$ Fisher & $<0.001 * *$ \\
\hline $\begin{array}{l}\text { Complication of type } 2 \text { diabetes mellitus } \\
\text { - Incorrect answer (11) } \\
\text { - Correct answer (91) } \\
\end{array}$ & $\begin{array}{c}6 \\
60 \\
\end{array}$ & $\begin{array}{c}54.5 \\
66 \\
\end{array}$ & $\begin{array}{c}5 \\
31 \\
\end{array}$ & $\begin{array}{c}45.5 \\
34 \\
\end{array}$ & ${ }^{\mathrm{a}}$ Fisher & 0.455 \\
\hline
\end{tabular}

${ }^{\mathrm{a}}$ Fisher's exact test ${ }^{\mathrm{b}}$ Chi square test

Table $(2)$ showed that there was highly statistical significance difference $\left(\mathrm{P}<0.001^{* *}\right)$ between family physicians and general practitioners regarding answers about routine care of diabetic patients, optimal HbA1c level diabetic patients, optimal level of bl. pressure for diabetic patients, times of ordering lipid profile, how many times to assess BMI, how can you examine feet. There was statistically significance difference $\left(\mathrm{P}<0.05^{*}\right)$ regarding when blood pressure was assessed, optimal level of lipid profile for diabetic patients, optimal level of BMI for diabetic patients, method to check nephropathy, time of ordering nephropathy check, if vaccinations recommended to diabetic patients, type of recommended vaccines with no statistically significance difference $(P \geq 0.05)$ regarding the remaining items. 
Table (3): Comparison between family physicians and general practitioners regarding practice about routine care of type 2 diabetes mellitus $(\mathrm{n}=102)$

\begin{tabular}{|c|c|c|c|c|c|c|}
\hline \multirow[t]{2}{*}{ Practice } & \multicolumn{2}{|c|}{$\begin{array}{c}\text { Family } \\
\text { physicians }(n=66)\end{array}$} & \multicolumn{2}{|c|}{$\begin{array}{c}\text { General } \\
\text { practitioners } \\
(\mathbf{n}=36) \\
\end{array}$} & \multirow[t]{2}{*}{ Test } & \multirow[t]{2}{*}{$P$ value } \\
\hline & No & $\%$ & No & $\%$ & & \\
\hline $\begin{array}{l}\text { HbA1c } \\
\text { - Not checked (10) } \\
\text { - Checked (92) }\end{array}$ & $\begin{array}{c}5 \\
61\end{array}$ & $\begin{array}{l}50 \\
66\end{array}$ & $\begin{array}{c}5 \\
31\end{array}$ & $\begin{array}{l}50 \\
34\end{array}$ & ${ }^{\mathrm{a}}$ Fisher & 0.306 \\
\hline $\begin{array}{l}\text { Blood pressure } \\
\text { - Not checked (9) } \\
\text { - Checked (93) } \\
\end{array}$ & $\begin{array}{c}3 \\
63 \\
\end{array}$ & $\begin{array}{c}33 \\
67.7 \\
\end{array}$ & $\begin{array}{c}6 \\
30 \\
\end{array}$ & $\begin{array}{c}67 \\
32.3 \\
\end{array}$ & ${ }^{\mathrm{a}}$ Fisher & $0.039 *$ \\
\hline $\begin{array}{l}\text { Foot examination } \\
\text { - Not checked ( } 8) \\
\text { - Checked (94) }\end{array}$ & $\begin{array}{c}2 \\
64\end{array}$ & $\begin{array}{l}25 \\
68\end{array}$ & $\begin{array}{c}6 \\
30\end{array}$ & $\begin{array}{l}75 \\
32\end{array}$ & ${ }^{\mathrm{a}}$ Fisher & $0.014 *$ \\
\hline $\begin{array}{l}\text { Eye examination } \\
\text { - Not checked (11) } \\
\text { - Checked (91) } \\
\end{array}$ & $\begin{array}{c}4 \\
62 \\
\end{array}$ & $\begin{array}{c}36.4 \\
68 \\
\end{array}$ & $\begin{array}{c}7 \\
29 \\
\end{array}$ & $\begin{array}{c}63.6 \\
32 \\
\end{array}$ & ${ }^{\mathrm{a}}$ Fisher & $0.037^{*}$ \\
\hline $\begin{array}{l}\text { Kidney function test } \\
\text { - Not checked (13) } \\
\text { - Checked (89) } \\
\end{array}$ & $\begin{array}{c}4 \\
62 \\
\end{array}$ & $\begin{array}{r}30.8 \\
69.7 \\
\end{array}$ & $\begin{array}{c}9 \\
27 \\
\end{array}$ & $\begin{array}{l}69.2 \\
30.3 \\
\end{array}$ & ${ }^{\mathrm{a}}$ Fisher & $0.006^{*}$ \\
\hline $\begin{array}{l}\text { Blood lipids } \\
\text { - Not checked (16) } \\
\text { - Checked (86) }\end{array}$ & $\begin{array}{c}7 \\
59 \\
\end{array}$ & $\begin{array}{r}43.8 \\
68.6 \\
\end{array}$ & $\begin{array}{c}9 \\
27\end{array}$ & $\begin{array}{l}56.2 \\
31.4 \\
\end{array}$ & b3.649 & 0.056 \\
\hline $\begin{array}{l}\text { Weight (BMI) } \\
\text { - Not checked (10) } \\
\text { - Checked (92) }\end{array}$ & $\begin{array}{c}5 \\
61\end{array}$ & $\begin{array}{l}50 \\
66\end{array}$ & $\begin{array}{c}5 \\
31\end{array}$ & $\begin{array}{l}50 \\
34\end{array}$ & ${ }^{\mathrm{a}}$ Fisher & 0.306 \\
\hline $\begin{array}{l}\text { Waist circumference } \\
\text { - Not checked (62) } \\
\text { - Checked (40) } \\
\end{array}$ & $\begin{array}{l}35 \\
31 \\
\end{array}$ & $\begin{array}{l}56.5 \\
77.5 \\
\end{array}$ & $\begin{array}{c}27 \\
9 \\
\end{array}$ & $\begin{array}{r}43.5 \\
22.5 \\
\end{array}$ & b4.717 & $0.030^{*}$ \\
\hline $\begin{array}{l}\text { Healthy eating review } \\
\text { - Not checked (8) } \\
\text { - Checked (94) }\end{array}$ & $\begin{array}{c}3 \\
63 \\
\end{array}$ & $\begin{array}{c}37.5 \\
67 \\
\end{array}$ & $\begin{array}{c}5 \\
31 \\
\end{array}$ & $\begin{array}{c}62.5 \\
33 \\
\end{array}$ & ${ }^{\mathrm{a}}$ Fisher & 0.093 \\
\hline $\begin{array}{l}\text { Physical activity review } \\
\text { - Not checked (6) } \\
\text { - Checked (96) } \\
\end{array}$ & $\begin{array}{c}2 \\
64 \\
\end{array}$ & $\begin{array}{l}33 \\
67 \\
\end{array}$ & $\begin{array}{c}4 \\
32 \\
\end{array}$ & $\begin{array}{l}67 \\
33 \\
\end{array}$ & ${ }^{\mathrm{a}}$ Fisher & 0.097 \\
\hline $\begin{array}{l}\text { Medication review } \\
\text { - Not checked (3) } \\
\text { - Checked (99) } \\
\end{array}$ & $\begin{array}{l}0.0 \\
66 \\
\end{array}$ & $\begin{array}{c}00 \\
66.7 \\
\end{array}$ & $\begin{array}{c}3 \\
33 \\
\end{array}$ & $\begin{array}{r}100 \\
33.3 \\
\end{array}$ & ${ }^{\mathrm{a}}$ Fisher & $0.017^{*}$ \\
\hline $\begin{array}{l}\text { Smoking cessation } \\
\text { - Not checked (34) } \\
\text { - Checked (68) }\end{array}$ & $\begin{array}{l}13 \\
53 \\
\end{array}$ & $\begin{array}{l}38 \\
78 \\
\end{array}$ & $\begin{array}{l}21 \\
15 \\
\end{array}$ & $\begin{array}{l}62 \\
22 \\
\end{array}$ & ${ }^{\mathrm{b}} 15.65$ & $<0.001 * *$ \\
\hline $\begin{array}{l}\text { Diabetes self-management } \\
\text { - Not checked (20) } \\
\text { - Checked (82) }\end{array}$ & $\begin{array}{l}10 \\
56\end{array}$ & $\begin{array}{c}50 \\
68.3\end{array}$ & $\begin{array}{l}10 \\
26\end{array}$ & $\begin{array}{c}50 \\
31.7\end{array}$ & b2.356 & 0.125 \\
\hline $\begin{array}{l}\text { Emotional health } \\
\text { - Not checked (3) } \\
\text { - Checked (99) } \\
\end{array}$ & $\begin{array}{c}0.0 \\
66 \\
\end{array}$ & $\begin{array}{c}00 \\
66.7 \\
\end{array}$ & $\begin{array}{c}3 \\
33 \\
\end{array}$ & $\begin{array}{r}100 \\
33.3 \\
\end{array}$ & ${ }^{\mathrm{a}}$ Fisher & $0.017^{*}$ \\
\hline
\end{tabular}

${ }^{\mathrm{a}}$ Fisher's exact test ${ }^{\mathrm{b}}$ Chi square test.

Table (3) showed that there was highly statistical significance difference ( $\mathrm{P}<0.001 * *)$ between family physicians and general practitioners regarding checking of smoking cessation and statistically significance difference $\left(\mathrm{P}<0.05^{*}\right)$ regarding blood pressure, foot examination, eye examination, kidney function test, waist circumference, medication review and emotional health. There was no statistically significance difference $(\mathrm{P}>0.05)$ regarding the remaining items of practice about routine care of type 2 diabetes mellitus. 


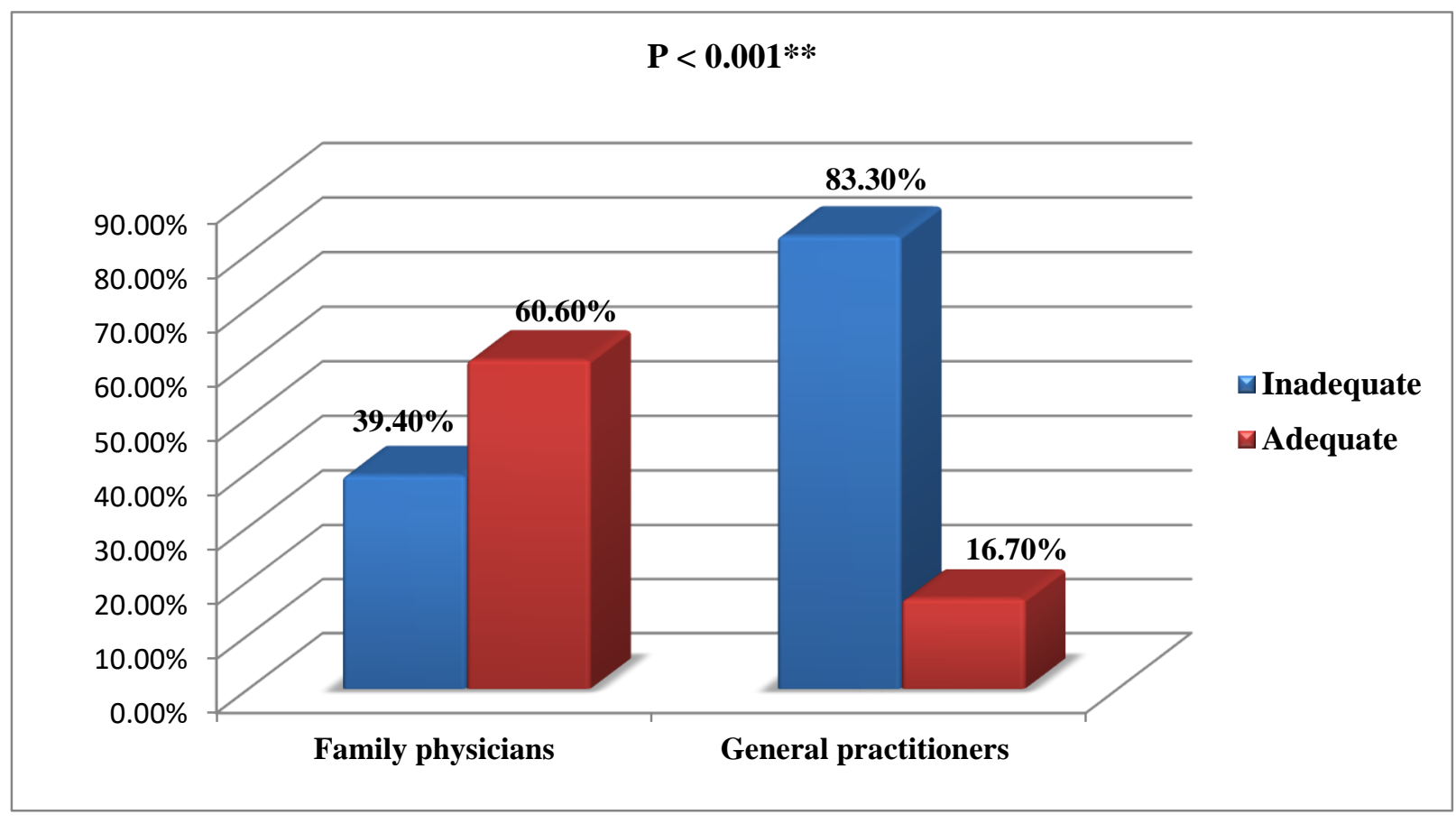

Figure (3): Comparison between family physicians and general practitioners regarding total score of practice about routine care of type 2 diabetes mellitus $(n=102)$

Figure (3) showed that there was highly statistical significance difference $(\mathrm{P}<0.001 * *)$ between family physicians and general practitioners regarding total practice score about routine care of type 2 diabetes mellitus.

\section{DISCUSSION}

As revealed from the current study, the majority of the studied doctors were females. About two thirds of them had family medicine postgraduate degree (scientific degree). More than half of them had period of experience more than 4 years, and most of them attended scientific courses.

In the starting of consultations, all doctors were welcoming friendly with the patients. This is inconsistent with Mercer $\boldsymbol{e t} \boldsymbol{a l} .{ }^{(5)}$ who found about onehalf of studied doctors had friendly welcoming.

Regarding consultation privacy, the current study found that about one half of the studied doctors were insured privacy during consultation. This disagrees with Dearden $\boldsymbol{e t} \boldsymbol{a l} .{ }^{\left({ }^{(6)}\right.}$ who found that ninety percentage of doctors insured privacy, and with Rethans et al. ${ }^{(7)}$ who found that about two thirds of doctors in general practice in the Netherlands insured privacy. In addition, with Gadallah et al. ${ }^{\left({ }^{(8)}\right.}$ who found that two thirds of doctors in two Health Centers in Egypt insured privacy? Low percent of ensuring privacy in present study during consultations was mainly due to overcrowding of clinics, and lack of adequate places and rooms in health centers where there was more than one doctor in the same clinic and every one of them interact with a patient at the same time.

The current study revealed that about one half of studied doctors were encouraging patients to ask questions. This is in agreement with Mercer et al. ${ }^{(5)}$ who found that about one half of their studied doctors encourage patients to ask questions. Encouraging the patient to ask questions is not only a method of information seeking, but also a mechanism of patient participation (patient-centered care) and verifying understanding. It allows the patient's point of view to guide the conversation, which has been shown to be positively associated with health outcomes ${ }^{(9)}$.

The majority of studied doctors maintain gesture to continue with patients during consultation. This is in contrast with Collins et al. ${ }^{(\mathbf{1 0})}$ who found that one third of their studied doctors maintained gestures to continue.

As revealed from the current study about one-half of studied doctors emphasized on understanding and follow up. This disagrees with Mercer $\boldsymbol{e t}$ al. ${ }^{(5)}$ who found that two thirds of their studied doctors emphasized on understanding and follow up.

The current study found that the majority of doctors asked about symptoms of diabetes. This agrees with Theban and Bajaba (11) study, which was conducted on family physicians to assess their attitudes and practices in managing people with type II diabetes mellitus and the majority of them were aware about the different symptoms of diabetes and asked about it.

Physical examination is a very important part of diabetes care to detect any complication early and to assess the general condition of diabetic patient. In this regards, the present study showed that two thirds of studied doctors examined patients. This agrees with Kevin Krane et al. ${ }^{\left({ }^{12}\right)}$ who found that about three quarters of studied doctors examine the patients physically.

Doctors should be careful to take a good medical history including diet compliance, physical activity, medicine use, and drugs adverse effects because these 
reduce patients' compliance ${ }^{(\mathbf{1 4})}$. Assessment of doctors' practice regarding asking about diet compliance revealed that the majority of them had optimal performance level. Our results are consistent with results of a study by Chwalow $\boldsymbol{e t}$ al. ${ }^{(15)}$ which displayed that family physicians and general practitioners had favorable attitude towards monitoring of blood sugar level, foot care, regular follow up of diabetic patients, diet counseling and physical activity. This difference was mainly because most of our diabetic patients were obese and we had many bad dietary habits in Egypt and Arabian countries, which make the doctors interest in dietary compliance of their patients.

As regards inquiring about physical activities, the present study showed that about three quarters of studied doctors had optimal practice. This is in agreement with Foster $\boldsymbol{e t} \boldsymbol{a l} .{ }^{(16)}$ who reported that less than half of the studied cases inquired about physical activities.

Misbelieves must be removed by patient education as it may lead to noncompliance to treatment in years to come looking at the chronicity of disease, in a country like India where nearly $85-90 \%$ type 2 diabetics are noncompliant. Nearly, $40 \%$ were unaware about asymptomatic nature and polyphagia was not known to them despite having the disease for on average 9 years. The highest lacunae in knowledge was with regard to insulin and type 2 diabetes, where nearly half were not aware about insulin level in their type of diabetes misinterpreting insulin injection as harmful for the body (18).

The current study found that little number of studied doctors asked about adverse effects of medication. This is in agreement with Kahf et al. ${ }^{(19)}$ who stated that most of the studied doctors asked about adverse effects of medication and were aware about it.

As regards to clinical care providing, about onehalf of studied doctors had optimal practical skills in providing care for diabetic patients. The majority of studied doctors especially family physicians had optimal practical skills regarding asking about smoking in consultations. This is in agreement with Kevin Krane et al. ${ }^{(\mathbf{1 2})}$ who reported that the majority of their studied doctors asked about smoking habits. Egyptian doctors neglected to ask about smoking due to religious reasons for both genders. It could also be related to cultural beliefs about behavior appropriate to each gender, such as alcohol consumption and smoking being regarded as natural for men, but not for women ${ }^{(20)}$.

As revealed from current study, doctors' age and scientific degree affect their knowledge and practice. All family physicians were perfect in their practice toward type 2 diabetic patients more than general practitioner was. This disagrees with Niroomand et al. (21) who investigated the Iranian physicians' KAP on diabetes mellitus and its management. Subjects' age and time since graduation in general medicine and specialty were inversely correlated by knowledge and practice.
Therefore, physicians with older age seems to be in priority for educational programs.

The current study revealed that there was no association between doctor's sex and practice. This finding is supported by one large study on diabetes in primary care in USA ${ }^{(22)}$. However, in other studies female physicians in primary care generally communicate in a more patient-centered way than male physicians ${ }^{(23)}$.

The present study revealed that there was no association between doctor's age and practice, which agrees with Shuval et al. ${ }^{(24)}$ who found that no role for doctor's age in doctor-patient relationship. However, other researches showed a preference for older doctors who have more experience in communication (25). Others showed a preference for younger doctors ${ }^{(26)}$.

The present study revealed that there was no association between doctors' period of experience and their practice, and there was no association between their period of experience and each item of performance, which disagrees with Kahf et al. ${ }^{\left({ }^{(19)}\right.}$. They stated that family physicians with relevant certifications or previously enrolled in relevant training courses had significantly higher knowledge regarding diagnosis of diabetes when compared to their colleagues who neither certified nor enrolled in such courses.

The current study revealed that there was no association between doctors' attendance of courses and their practice, and there was no association between their attendance of courses and each item of practice. A study of Peimani $\boldsymbol{e t}$ al. ${ }^{(27)}$ revealed the presence of a correlation between the evidence-based knowledge of primary care physicians and the quality of care they provide. Another study by Fogelman et al. ${ }^{(28)}$ showed that board certified family physicians and their residents tended to be younger and to exhibit greater knowledge of diabetes than did non-BCFPs. Another study in Saudi Arabia by Aldarbi et al. ${ }^{(29)}$ reported that primary care physicians who attended educational courses or training workshops on DM had significantly better knowledge and practices grades than those who did not.

There was no-association between the studied doctors' socio-demographic characteristics and their practice that was mainly due to homogenous sample (most of doctors were females, from Zagazig University and had family medicine post-graduate degree with a period of experience more than 4 years, and attended family medicine courses). Also, may be because all of them worked in a similar environment of work, and with similar resources.

The current study found that slightly more than one-half of studied doctors had optimal practical skills as regards communication skills with diabetic patients.

The present study had some limitations including time, which was limited for the doctors to fill out the questionnaire, so some doctors were uncooperative with me. Long distance between health units and centers present in Zagazig and villages around it was a hindrance also. 


\section{CONCLUSION}

The doctors were good in communication skills with patients, describing medications, emphasizing on blood sugar control. However, they were not good in measuring waist circumference and knowledge about time to check patients' feet.

\section{RECOMMENDATION}

Periodic diabetes management training courses should be organized for doctors in Family Health Centers to increase the quality of their practice toward diabetes type 2 patients. Also, regulations, policies, guidelines and the needed for modifications should be revised to improve doctors' practice toward type 2 diabetic patients.

Financial support and sponsorship: Nil.

Conflict of interest: Nil.

\section{REFERENCES}

1. ADA (2021): Suggested citation: American Diabetes Association. 3. Prevention or delay of type 2 diabetes: Standards of Medical Care in Diabetes-2021. Diabetes Care, 44 (1): 34-39.

2. Al-Maskari F, El-Sadig M, Al-Kaabi J et al. (2013): Knowledge, attitude and practices of diabetic patients in the United Arab Emirates. PloS One, 8 (1): 52857-61.

3. Alakhali K, Mohammad A (2014): "Prescribing pattern of antibiotics in pediatric patients in the Jazan Region, Kingdom of Saudi Arabia," RGUHS Journal of Pharmaceutical Sciences, 4: 120-124.

4. Kenny D, Veldhuijzen $W$, LeBlanc $T$ et al. (2010): Interpersonal perception in the context of doctor-patient communication. Social Science \& Medicine, 70: 763-768.

5. Mercer L, Tanabe P, Pang P et al. (2008): Patient perspectives on communication with the medical team: Pilot study using the communication assessment tool-team (CAT-T): Patient Educ. Counsel, 73: 220-223.

6. Dearden A, Smithers M, Thapar A (2006): Interruptions during general practice consultations--the patients' view. FamPract., 13 (2): 166-169.

7. Rethans J, Hoppener P, Wolfs G et al. (2008): Do personal computers make doctors less personal? Br Med J (Clin Res Ed). 296 (6634): 1446-1448.

8. Gadallah M, Zaki B, Rady M et al. (2003): Patient satisfaction with primary health care services in two districts in Lower and Upper Egypt. East Mediterr Health J., 9 (3): 422-430.

9. Travaline J, Ruchinskas R, D'Alonzo G (2005): Patientphysician communication: why and how. J Am Osteopath Assoc., 105 (1): 13-18.

10. Collins L, Schrimmer A, Diamond J et al. (2010): Evaluating verbal and non-verbal communication skills, in an ethnogeriatric OSCE. Patient Educ. Counsel, 3706: 1-5.

11. Theban A, Bajaba M (2019): Knowledge, attitude and practice regarding diabetes among Saudi MOH Primary Healthcare Physicians in Jeddah: A Cross Sectional Study. Int J Med Res Prof., 5 (1): 192-201.

12. Krane N, Anderson D, Lazarus C et al. (2009): Physician practice behavior and practice guidelines: using unannounced standardized patients to gather data. Journal of General Internal Medicine, 24 (1): 53-56.

13. Abdulhadi N, Al-Shafaee M, Östenson C et al. (2006): Quality of interaction between primary health-care providers and patients with type 2 diabetes in Muscat, Oman: an observational study. BMC Family Practice, 7 (1): 1-10.

14. Chaudhury A, Duvoor C, Reddy Dendi V et al. (2017): Clinical review of antidiabetic drugs: implications for type 2 diabetes mellitus management. Frontiers in Endocrinology, 8: 611.

15. Chwalow A, Costaghiola D, Mesbah M et al. (2016): Management of type 2 diabetes mellitus in France: Attitudes and practitioners among a representative sample of general practitioners. Diabetes Metab., 4: 458-464.

16. Foster T, Mowatt L, Mullings J (2016): Knowledge, Beliefs and Practices of Patients with Diabetic Retinopathy at the University Hospital of the West Indies Jamaica. J Community Health, 41 (3): $584-92$.

17. Kishore J, Kohli C, Gupta $\mathbf{N}$ et al. (2015): Awareness, practices and treatment seeking behavior of type 2 diabetes mellitus patients in Delhi. Ann Med Health Sci Res., 5: 266-73.

18. Lahiri S, Haldar D, Chowdhury $S$ et al. (2011): Junctures to the therapeutic goal of diabetes mellitus: Experience in a tertiary care hospital of Kolkata. J Midlife Health, 2: 31-36.

19. Kahf M, Ayad K, Gabr M et al. (2020): Knowledge, Attitudes and Practices of Primary Health Care Physicians Regarding Diabetes Management: A Cross Sectional Study in Gharbia Governorate, Egypt. Asian Journal of Medicine and Health, 20: $1-15$.

20. Kakade A, Mohanty I, Rai S (2016): Assessment of knowledge, attitude and self-care activities among type-2 diabetic patients attending a tertiary care teaching hospital. Int $\mathrm{J}$ Basic Clin Pharmacol., 5: 2458-62.

21. Niroomand M, Ghasemi S, Karimi-Sari $H$ et al. (2017): Knowledge, attitude, and practice of Iranian internists regarding diabetes: A cross-sectional study. Diabetes Metab., 41 (3): 179186.

22. Kim C, McEwen L, Gerzoff $R$ et al. (2005): Is physician gender associated with the quality of diabetes care? Diabetes Care, 28 (7): 1594-1598.

23. Roter D, Hall J (2006): Physician gender and patient-centered communication: a critical review of empirical research. Annu Rev Public Health, 25: 497-519.

24. Shuval K, Linn S, Brezis M et al. (2010): Association between primary care physicians' evidence-based medicine knowledge and quality of care. International Journal for Quality in Health Care, 22 (1): 16-23.

25. Kite M, Deaux K, Miele M (2011): Stereotypes of young and old, does age outweigh gender? Psych Ageing, 6: 19-27.

26. Shaha R, Ogden J (2006): 'What's in a face? The role of doctor ethnicity, age, gender in the formation of patients' judgements, an experimental study. Patient Educ Counsel., 60: 136-141.

27. Peimani M, Tabatabaei $M$, Heshmat $R$ et al. (2010): Knowledge, attitude and practice of physicians in the field of diabetes and its complications, a pilot study. Iran J Diabetes Lipid Disord., 9: 1-7.

28. Fogelman Y, Goldfracht M, Karkabi K (2015): Managing Diabetes Mellitus: A Survey of Attitudes and Practices Among Family Physicians. J Community Health, 40 (5): 1002-1007.

29. Aldarbi M, Alsaleem M, Alsaleem $S$ et al. (2018): The variance of knowledge and practices about diabetes mellitus in primary health care physicians of Jazan region, Kingdom of Saudi Arabia. Biomedical Research, 29 (10): 2083-2089. 